\title{
Gold Nanoparticles Conjugation Enhances Antiacanthamoebic Properties of Nystatin, Fluconazole and Amphotericin B
}

\author{
Ayaz Anwar ${ }^{1}$, Ruqaiyyah Siddiqui ${ }^{1}$, Muhammad Raza Shah ${ }^{2}$, and Naveed Ahmed Khan ${ }^{1 *}$ \\ ${ }^{1}$ Department of Biological Sciences, School of Science and Technology, Sunway University, Subang Jaya 47500, Selangor, Malaysia \\ ${ }^{2}$ International Center for Chemical and Biological Sciences, H.E.J. Research Institute of Chemistry, University of Karachi, Karachi 75270, \\ Pakistan
}

\author{
Received: May 17, 2018 \\ Revised: October 29, 2018 \\ Accepted: November 1, 2018 \\ First published online \\ November 1, 2018 \\ *Corresponding author \\ Phone: +603-74918622; \\ Fax: +603-56358630; \\ E-mail: naveed5438@gmail.com
}

pISSN 1017-7825, eISSN 1738-8872

Copyright(C) 2019 by

The Korean Society for Microbiology and Biotechnology
Parasitic infections have remained a significant burden on human and animal health. In part, this is due to lack of clinically-approved, novel antimicrobials and a lack of interest by the pharmaceutical industry. An alternative approach is to modify existing clinically-approved drugs for efficient delivery formulations to ensure minimum inhibitory concentration is achieved at the target site. Nanotechnology offers the potential to enhance the therapeutic efficacy of drugs through modification of nanoparticles with ligands. Amphotericin B, nystatin, and fluconazole are clinically available drugs in the treatment of amoebal and fungal infections. These drugs were conjugated with gold nanoparticles. To characterize these goldconjugated drug, atomic force microscopy, ultraviolet-visible spectrophotometry and Fourier transform infrared spectroscopy were performed. These drugs and their gold nanoconjugates were examined for antimicrobial activity against the protist pathogen, Acanthamoeba castellanii of the T4 genotype. Moreover, host cell cytotoxicity assays were accomplished. Cytotoxicity of these drugs and drug-conjugated gold nanoparticles was also determined by lactate dehydrogenase assay. Gold nanoparticles conjugation resulted in enhanced bioactivity of all three drugs with amphotericin B producing the most significant effects against Acanthamoeba castellanii $(p<0.05)$. In contrast, bare gold nanoparticles did not exhibit antimicrobial potency. Furthermore, amoebae treated with drugs-conjugated gold nanoparticles showed reduced cytotoxicity against HeLa cells. In this report, we demonstrated the use of nanotechnology to modify existing clinically-approved drugs and enhance their efficacy against pathogenic amoebae. Given the lack of development of novel drugs, this is a viable approach in the treatment of neglected diseases.

Keywords: Acanthamoeba, antimicrobial, gold nanoparticles, Amphotericin B, nystatin, fluconazole

\section{Introduction}

Nanomedicine has recently emerged as a viable alternative to organic drugs especially in the improvement of new and more efficacious antimicrobials [1, 2]. Due to a continuous lack in-drug approvals, and ever-growing resistance of microbes against common antimicrobial drugs, nanoconjugates are anticipated as the next generation of antimicrobial agents [3]. Nanoparticles are effective drug delivery agents and can increase the bioavailability of drugs at target sites.
Due to their small size, they can be loaded with a high amount of drug molecules, thus reducing the risks of toxicity and side effects. Numerous metallic and metal oxide nanoparticles now being utilized in biomedicines have been developed with copper, silver, gold, platinum, iron oxide, zinc oxide, etc. These nanoparticles have shown tremendous diagnostic and therapeutic potential against cancer, conditions of the central nervous system, and infectious disease. The most common examples of nanoparticles used against infectious diseases are of silver 
and gold due to their biocompatibility. Gold nanoparticles have been associated with enhancing bioactivity and reducing the cytotoxicity of antimicrobial drugs [4, 5], while also improving the bioavailability of drugs by through better drug delivery systems [6].

Acanthamoeba is an opportunistic free-living amoeba, and a causative agent of a rare but fatal central nervous system infection: granulomatous amoebic encephalitis (GAE), and a blinding eye infection, recognized as Acanthamoeba keratitis [7-9]. Currently there are none or very limited options for treatment and management of Acanthamoeba infections. These include a combination of drugs such as biguanides, amidine derivatives, and azoles; nonetheless these drugs suffer from some serious drawbacks such as long treatment plans and unspecific toxic effects on host cells [10-12].

Macrocyclic polyenes such as Amphotericin B and nystatin, and fluconazole target the ergosterol pathway to rupture the plasma membrane. As ergosterol is a component of the Acanthamoeba plasma membrane, it is a logical target to develop drugs against Acanthamoeba infections, however owing to their cytotoxicity to host cells; ergosteroltargeting drugs are in limited use clinically [13-15]. In our recent report, silver nanoparticles conjugated with these drugs were shown to exhibit significant amoebicidal effects, while their host cell cytotoxicity was also reduced as compared to drugs alone [16].

In our present study, fluconazole, nystatin, and amphotericin B were conjugated with gold nanoparticles (AuNPs). These nanoparticles were produced by silver nitrate reduction through sodium borohydride alongside the drugs. Fourier-transformation infrared (FT-IR) spectroscopy, atomic force microscopy (AFM) and ultraviolet-visible (UVvis) spectrophotometry were accomplished to characterize drugs, and drugs joined together with nanoparticles. Subsequently, their antiamoebic activities against $A$. castellanii were determined.

\section{Materials and Methods}

\section{Chemicals}

For drug synthesis, chemicals of analytical grade were utilized. Sodium borohydride and tetrachloroauric acid were acquired from Merck. Nystatin, fluconazole and amphotericin B were obtained from Sigma-Aldrich.

\section{Synthesis of AuNPs}

AuNPs were produced by using sodium borohydride as a reducing agent in the presence of drugs [17]. Nystatin, fluconazole and amphotericin B coated with tetrachloroauric acid were reacted at $0.1 \mathrm{mM}$ concentration in different volume ratios to obtain stable gold nanoparticles. Briefly, Amp-AuNPs were obtained at 1:1 volume ratio, followed by addition of $30 \mu \mathrm{l}$ of $4 \mathrm{mM}$ sodium borohydride aqueous solution (prepared freshly). A color change from light yellow to ruby red of the reaction concoction signified reduction of gold ions and the establishment of Amp-AuNPs. This reaction mixture was further agitated for $1 \mathrm{~h}$ until no change in color was detected. The process was repeated for Nys-AuNPs and Flu-AuNPs, by enhancing various v/v ratios of gold solution in the presence of the drugs. To attain stable NysAuNPs and Flu-AuNPs, a respective gold-to-drug volume ratio of 5:1 was utilized. AuNPs alone were produced using the same method but with a lack of drugs or stabilizing agents. Subsequently, to remove large aggregates, any additional unbound drugs or side products from the colloidal suspension, centrifugation at $10,000 \times g$ for $10 \mathrm{~min}$ was done. Following nanoparticle synthesis, FT-IR spectroscope (Vector 22, Bruker) and UV-vis spectrophotometer (Evolution 300, Thermo Scientific) analysis was achieved. For morphological examination an AFM (Agilent 5500) was utilized as described previously [16].

\section{Acanthamoeba Cultures}

Acanthamoeba castellanii from the T4 genotype (ATCC 50492) were cultivated in $75 \mathrm{~cm}^{2}$ tissue culture flasks encompassing $10 \mathrm{ml}$ PYG medium or proteose peptone $0.75 \%(\mathrm{w} / \mathrm{v})$, glucose $1.5 \%$ $(\mathrm{w} / \mathrm{v})$, yeast extract $0.75 \%(\mathrm{w} / \mathrm{v})$ and maintained at $30^{\circ} \mathrm{C}[18]$. Trophozoites adhered to the surface of the flask were procured by washing with fresh PYG media followed by resuspension in $10 \mathrm{ml}$ RPMI-1640 and placing on ice for $15 \mathrm{~min}$ along with gentle shaking for 5 min. Next, the A. castellanii RPMI-1640 suspension was relocated to a $50 \mathrm{ml}$ tube, and centrifuged at 2,500 $\times g$ for $10 \mathrm{~min}$ and the pellet was resuspended in $1 \mathrm{~mL}$ RPMI-1640. A. castellanii were enumerated by means of a haemocytometer and subsequently utilized for assays.

\section{Amoebicidal Assay}

The amoebicidal activities of drugs alone, drugs joined together to AuNPs, and AuNPs alone were elucidated as reported earlier [18]. Briefly, $5 \times 10^{5}$ A. castellanii cells were incorporated with various $(5$ and $10 \mu \mathrm{M})$ concentrations of drugs alone or drugs conjugated with AuNPs, nanoparticles alone, and solvent controls at $30^{\circ} \mathrm{C}$ for $24 \mathrm{~h}$. Following this, Trypan blue cell exclusion assay was carried out. For controls, RPMI-1640 alone served as a negative control and $10 \mu \mathrm{M}$ of the anti-amoebic drug Chlorhexidine was utilized as a positive control. To elucidate the viability of amoebae, $0.1 \%$ Trypan blue was added and living (non-stained) A. castellanii were enumerated with a haemocytometer. Results obtained were presented as percentage inhibition: mean inhibition \pm standard error of three independent experiments performed in duplicate. The percentage of inhibition was calculated as follows: $\%$ inhibition $=100-$ (number of viable Acanthamoeba in sample treated/number of viable Acanthamoeba untreated) $\times 100$. 


\section{Henrietta Lacks Cervical Adenocarcinoma Cells (HeLa) Cells Culture}

Henrietta Lacks (HeLa) cells were typically cultivated using $75 \mathrm{~cm}^{2}$ culture flasks in RPMI-1640 comprising 10\% Nu-serum, $10 \%$ FBS, $1 \mathrm{mM}$ pyruvate, $2 \mathrm{mM}$ glutamine, streptomycin $(100 \mu \mathrm{g} / \mathrm{ml})$, penicillin $(100 \mathrm{Units} / \mathrm{ml})$, non-essential amino acids and vitamins. Prior to experiments, media were removed and $2 \mathrm{ml}$ of trypsin was added to detach cells from confluent flasks. Next, cells were centrifuged at 2,000 $\times g$ for $5 \mathrm{~min}$. The resulting pellet was resuspended in $30 \mathrm{ml}$ media, and harvested into a 96-well plate, incubated at $37^{\circ} \mathrm{C}$, with $95 \%$ humidity and $5 \% \mathrm{CO}_{2}$ for at least $24 \mathrm{~h}$. Plates were scrutinized using a light microscope until a visible uniform monolayer of HeLa cells was observed for use in the cytotoxicity assay.

\section{Cytotoxicity Assays}

To determine in vitro cytotoxicity of drug-conjugated AuNPs, cytotoxicity assays were performed using HeLa cells. Briefly, cells were developed until confluent in 24-well plates and different concentrations of drug nanoconjugates were added. The plates were placed at $37^{\circ} \mathrm{C}$ in a $5 \% \mathrm{CO}_{2}$ incubator for $24 \mathrm{~h}$. Supernatants were collected and the percentage cell cytotoxicity was elucidated by measuring lactate dehydrogenase release (Roche Applied Science). Cells alone represented the negative control, whereas HeLa monolayers completely lysed with $1 \%$ Triton X-100 represented the positive control corresponding to $100 \%$ cell death. Absorbance of each sample was recorded on a plate reader at $490 \mathrm{~nm}$. The percentage cytotoxicity was obtained: $\%$ cytotoxicity $=($ sample absorbance-negative control absorbance)/(positive control absorbance - negative control absorbance) $\times 100$. Acanthamoebafacilitated HeLa cell cytotoxicity was assessed as formerly depicted [19, 20]. Concisely, experiments were accomplished in 96-well plates containing uniform HeLa monolayers. Acanthamoeba $\left(1 \times 10^{4}\right)$ were treated with 5 and $10 \mu \mathrm{M}$ concentrations of drugs alone, drug-conjugated AuNPs, AuNPs alone, chlorhexidine $(10 \mu \mathrm{M})$, and incubated for $2 \mathrm{~h}$ at $37^{\circ} \mathrm{C}$. Moreover, centrifugation at 5,000 $\times g$ for $1 \mathrm{~min}$ was completed to eliminate any extracellular constituents, and the resulting pellet was resuspended in fresh RPMI. These test samples and controls were then added to HeLa

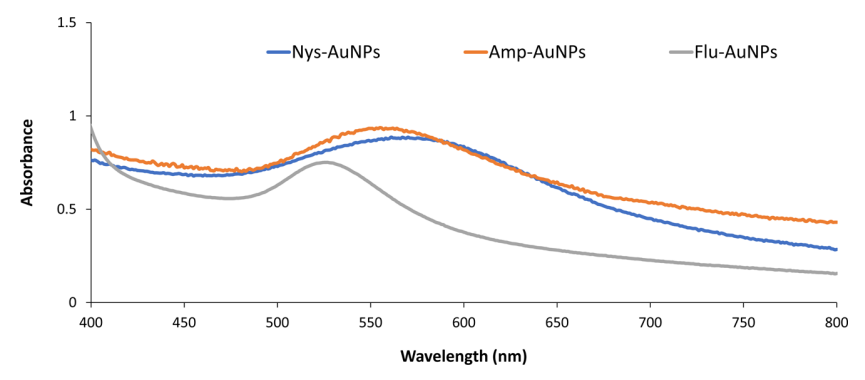

Fig. 1. UV-Vis spectra of drug-coated AuNPs showed surface plasmon resonance bands at 555, 561, and $525 \mathrm{~nm}$ for AmpAuNPs, Nys-AuNPs and Flu-AuNPs, respectively which indicates the successful formation of drug-conjugated AuNPs.

cell monolayers and incubated in a $5 \% \mathrm{CO}_{2}$ incubator for $24 \mathrm{~h}$ at $37^{\circ} \mathrm{C}$. Negative control values were obtained for monolayers in RPMI-1640 alone. Positive control values were acquired by completely lysing cells with $1 \%$ Triton X-100. Subsequently, the supernatants were carefully collected and cytotoxic activity elucidated by using the Lactate dehydrogenase (LDH) (Roche Applied Sciences) kit followed by absorbance estimation on a plate reader at $490 \mathrm{~nm}$. The percentage cytotoxicity was determined: $\%$ cytotoxicity $=$ (sample absorbance - negative control absorbance $) /$ (positive control absorbance - negative control absorbance) $\times 100$.

\section{Results}

\section{Drug-Coated Gold Nanoconjugates Characterized Using FT- IR Spectroscopy, UV-Vis, and AFM}

To characterize drug-coated AuNPs and to confirm the formation of nanoparticles, UV-vis spectrophotometry was performed. The characteristic surface plasmon resonance bands are shown in Fig. 1 with Amp-AuNPs, Nys-AuNPs and Flu-AuNPs corresponding to successful synthesis. The UV-vis spectra of Amp-AuNPs produced maximum absorption at $555 \mathrm{~nm}$, in comparison to Amphotericin B
Amp-AuNPs

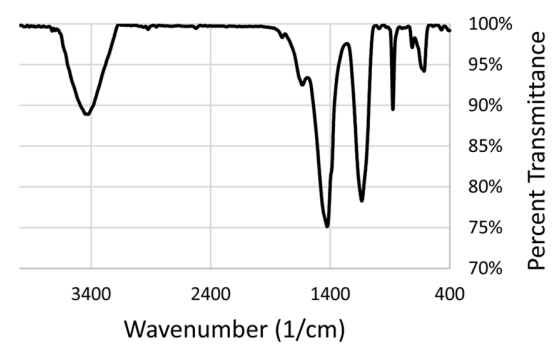

Nyst-AuNPs

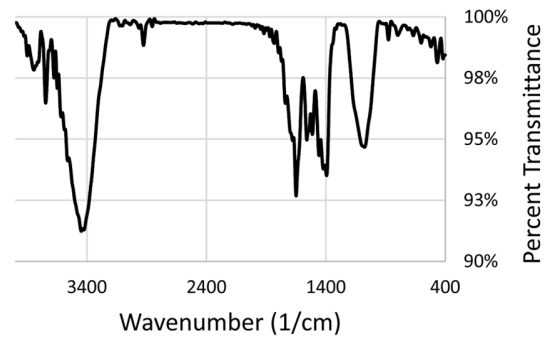

Flu-AuNPs

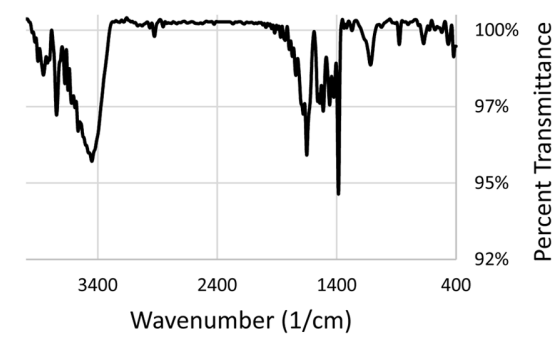

Fig. 2. FT-IR spectra of drug-conjugated AuNPs.

Hydroxyl groups stabilized AuNPs in the case of amphotericin B and nystatin, the whereas, triazole group chelated the AuNPs in Flu-AuNPs. Spectra were obtained by $\mathrm{KBr}$ disc method on Bruker Vector 22 instrument. 


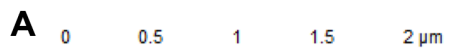

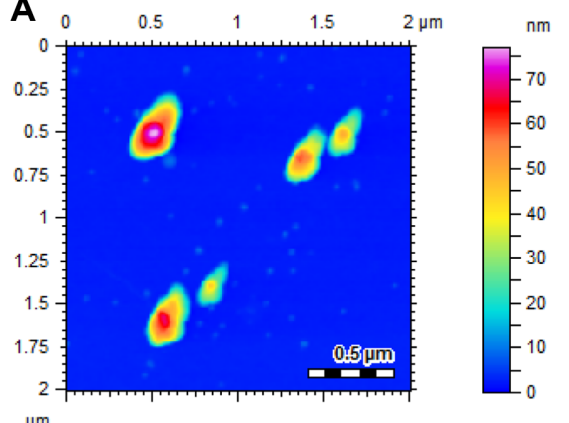

B 0

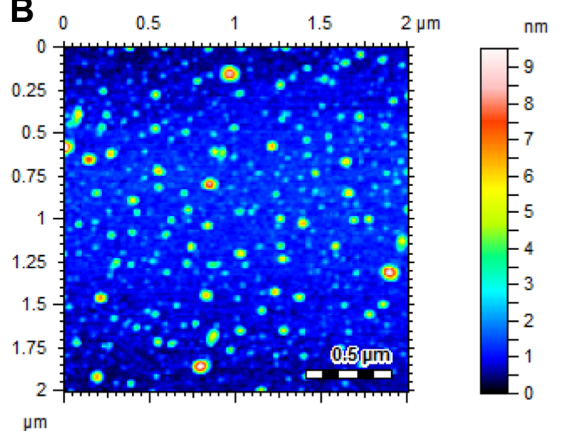

C

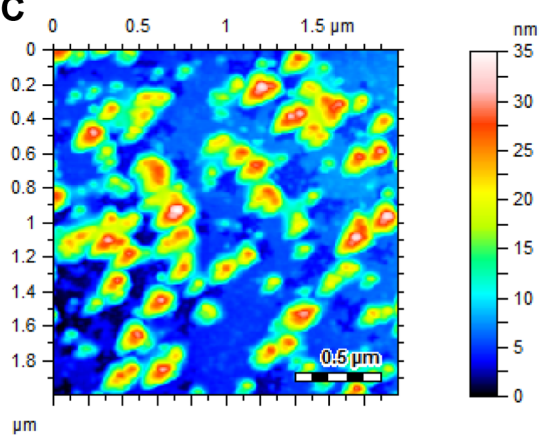

Fig. 3. AFM images of Amp-AuNPs; A, Nyst-AuNPs; B, Flu-AuNPs; C. Extensive imaging was done to describe representative images.

Nyst-AuNPs showed small particles size (10-50 nm), while Amp-AuNPs and Flu-AuNPs were found to be in the range of 50-200 nm.

alone, which reveals various absorption bands in the range of 280-400 nm [21], implying stabilized interactions with AuNPs. On the other hand, Nys-AuNPs and Flu-AuNPs presented a characteristic surface plasmon resonance (SPR) band for AuNPs at 561 and $525 \mathrm{~nm}$, respectively, associated with bands of approximately $280-330 \mathrm{~nm}$ and $260 \mathrm{~nm}$ correspondingly for fluconazole and nystatin alone [22, 23]. FT-IR analysis was achieved to report interaction between drugs and AuNPs (Fig. 2). Amphotericin B also showed a strong stretching band at 3,400 1/cm for hydrogen-bonded molecules and 1,645 1/cm for carbonyl, whereas, in AmpAuNPs these signals were shifted to 3,427 and 1,633 1/cm, respectively. Since, nystatin and amphotericin $B$ both have structural analogies, the same effects were observed in the case of Nyst-AuNPs. Fluconazole alone displayed an absorption band at $1,4131 / \mathrm{cm}$ portraying triazole ring stretching, which on the formation of Flu-AuNPs, shifted to $1,3841 / \mathrm{cm}$. These results indicate that the interaction of hydroxyl groups for amphotericin B and nystatin, and the triazole ring for fluconazole are responsible for the stabilization of AuNPs. The morphology and size of the nanoparticles were assessed by AFM. Nanoparticles including some larger aggregates were observed under AFM imaging (Fig. 3). Nyst-AuNPs provided the smallest particle size lying in the area of $10-50 \mathrm{~nm}$, while particle size was highly polydispersed for Amp-AuNPs and Flu-AuNPs, ranging from $50-200 \mathrm{~nm}$.

\section{AuNP-Conjugated Drugs Display Enhanced Amoebicidal Activity against $A$. castellanii in Comparison to Drugs Alone}

The amoebicidal potency of drugs alone and their gold

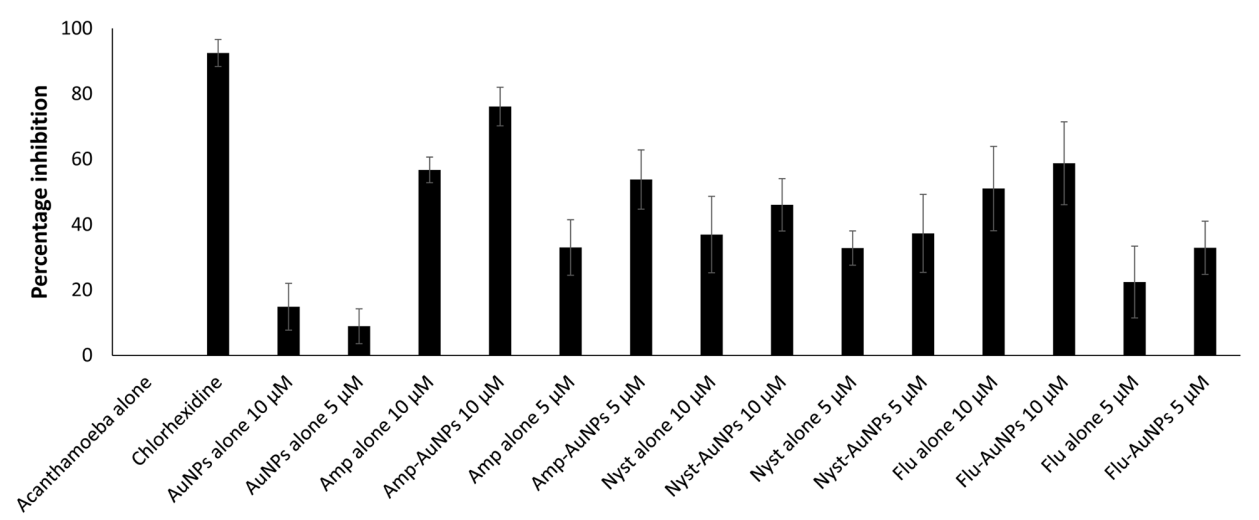

Fig. 4. A. castellanii viability was determined following incubation with various concentrations of drugs alone and drug conjugated AuNPs as described in Materials and Methods.

All three drugs conjugated AuNPs and drugs alone exhibited amoebicidal effects. Notably, Amp-AuNPs and Flu-AuNPs showed most prominent amoebicidal effects at $10 \mu \mathrm{M}$ compared with drugs alone ( $p<0.05$ using 2 sample T-test and two-tailed distribution). The results are presented as the mean inhibition \pm standard error of at least three independent experiments performed in duplicate. The percent inhibition was calculated as follows: \% inhibition $=100-($ number of viable Acanthamoeba in sample treated/number of viable Acanthamoeba untreated $) \times 100$. 


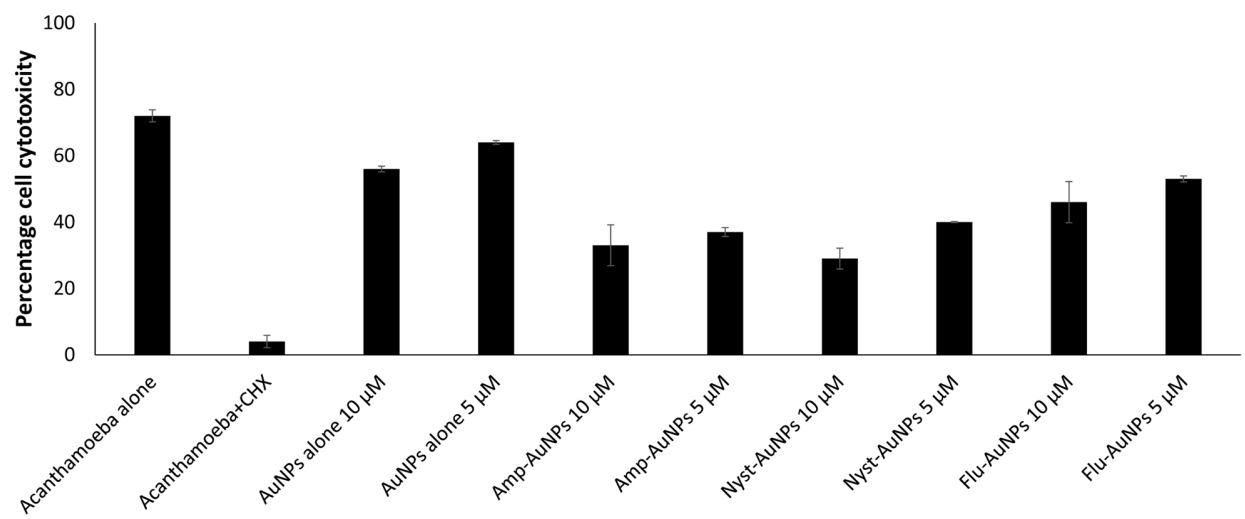

Fig. 5. Cytopathogenicity was determined by treating host cells with A. castellanii with and without pre-treatment with drugs and drug-coated AuNPs as described in Materials and Methods.

Untreated microbes killed more than $70 \%$ of cells, while microbes pre-treated with drugs and drug-coated AuNPs showed significantly reduced host cell cytotoxicity as compared to untreated microbes ( $p<0.05$ using 2 sample T-test and two-tailed distribution). The data are presented as means standard errors from at least three independent experiments performed in duplicate. The percent cytotoxicity was calculated as follows: \% cytotoxicity $=($ sample absorbance - negative control absorbance $) /($ positive control absorbance - negative control absorbance $) \times 100$.

nanoconjugates was determined by amoebicidal assay. All drugs exhibited significant amoebicidal activity at both 10 and $5 \mu \mathrm{M}$ concentrations except for fluconazole which only displayed amoebicidal results at $10 \mu \mathrm{M}(p<0.05$ by means of a 2-sample t-test and two-tailed distribution) (Fig. 4). Drug-coated AuNPs on the other hand, displayed more pronounced killing effects as compared to drugs alone. Amp-AuNPs exhibited amoebicidal results at $10 \mu \mathrm{M}$ with $76 \%$ inhibition as compared to $56 \%$ for amphotericin B alone. Similarly, at $5 \mu \mathrm{M}$ concentration, $11 \%$ increase was found in percent inhibition of Amp-AuNPs as compared to amphotericin B alone. Flu-AuNPs were also found to exhibit $11 \%$ increased percent inhibition at $5 \mu \mathrm{M}$ as compared to fluconazole alone which only showed $22 \%$ inhibition. However, no drastic changes were observed in the case of nystatin alone vs Nyst-AuNPs. On the contrary, treatment with AuNPs alone had no effect on amoebae inhibition.

\section{Cytopathogenicity of Drug-Conjugated AuNPs}

To ascertain if AuNP-conjugated drugs inhibit microbial cytopathogenicity against host cells, microbial mediated cytotoxicity assays were completed. When A. castellanii alone were incorporated with host cells this caused $72 \%$ cell cytotoxicity (Fig. 5). On the other hand, pre-treatment of $A$. castellanii with chlorhexidine resulted in minimal cell cytotoxicity. However, only Amp-AuNPs and Nyst-AuNPs reduced the $A$. castellanii cytotoxicity ( $p<0.05$ by means of a 2-sample $t$-test and two-tailed distribution). The cytotoxicity profile of drugs joined together with gold nanoparticles against HeLa cells was also evaluated. The results revealed that all drug-coated nanoparticles produced less than 25\% host cell death with the exception of fluconazole.

\section{Discussion}

Nanotechnology has recently emerged as a tremendous tool in the arena of biomedicine, particularly in diagnosis and drug delivery. Drug delivery carriers based on nanotechnology have been developed and are being utilized in clinical applications, such as in nanotubes, nanoparticles, and liposomes [24]. Nanomaterials play a pivotal role in drug delivery and provide numerous advantages including (i) augmented bioavailability; (ii) reduced side effects; (iii) precise drug targeting. Moreover, due to their miniature proportions, nanoparticles deliver a superior surface area for maximum drug packing in addition to elevated approachability for precise targets [25]. Numerous nanoparticles conjugated with drugs have been produced and utilized against several infectious diseases caused by resistant microbes and parasites [26]. In our recent study, we reported the enhanced effects of antifungal drugs nystatin, fluconazole amphotericin B conjugated with AgNPs on A. castellanii [16]. Since these drugs target the ergosterol pathway which is an essential component of the A. castellanii membrane, their conjugation with nanoparticles resulted in increased bioactivity. Here, we determined the effects of AuNP conjugation with these drugs on protist and human cells. Drug-coated AuNPs were created by the one-phase reduction method and 
categorized by UV-vis spectrophotometry, FT-IR spectroscopy, and AFM. All drug-coated AuNPs showed surface plasmon resonance (SPR) band in the range of $500-560 \mathrm{~nm}$, characteristic of medium-sized, ligand-stabilized AuNPs. FT-IR analysis showed the involvement of hydroxyl groups and triazole ring in the stabilization of nanoparticles. The sizes of nanoparticles were found to be in the range of 10$200 \mathrm{~nm}$, as a result of rapid reducing conditions. The conjugation of AuNPs to these drug molecules enhanced their antiacanthamoebic effects, however, the intravenous administration of these drugs lacks specificity and as a result causes host cell cytotoxicity. Conjugation with inert metal such as gold in the form of nanoparticles was hypothesized to decrease these side effects along with enhancement in the bioavailability of these drugs at low dosage.

The reason for the enhanced cytotoxicity of drugconjugated AuNPs is most probably due to enhanced transport of drugs to the target site. Recent literature reports on the antibacterial activity of nanoparticles suggest that their biocidal effects are highly dependent on their size, shape and surface modifications [27, 28]. Moreover, their mode of bacteria killing is supposed to be a combination of multiple factors including production of biocidal reactive oxygen species altering the DNA, interaction with thiolated amino acids of enzymes etc. [29, 30]. Hence nanoparticles are speculated as next-generation antibacterial agents due to their broad range of targets in microorganisms. In conclusion, these findings undoubtedly reveal that conjugation of drugs with nanoparticles improved and enhanced the antiamoebic activity of existing drugs. Future studies using in vivo models and the administration of drugs coated with nanoparticles via different portals of entry to assess their maximum biological potential need to be conducted.

\section{Acknowledgment}

The authors acknowledge Sunway University and HEJ Research Institute of Chemistry for their complete support.

\section{Conflict of Interest}

The authors have no financial conflicts of interest to declare.

\section{References}

1. Li X, Robinson SM, Gupta A, Saha K, Jiang Z, Moyano DF, et al. 2014. Functional gold nanoparticles as potent antimicrobial agents against multi-drug-resistant bacteria. ACS Nano 8: 10682-10686.

2. Yang X, Yang J, Wang L, Ran B, Jia Y, Zhang L, et al. 2017. Pharmaceutical Intermediate-modified gold nanoparticles: against multidrug-resistant bacteria and wound-healing application via electrospun scaffold. ACS Nano 11: 5737-5745.

3. Maincent P, Le Verge R, Sado P, Couvreur P, Devissaguet J-P. 1986. Disposition kinetics and oral bioavailability of vincamineloaded polyalkyl cyanoacrylate nanoparticles. J. Pharm. Sci. 75: 955-958.

4. Aqeel Y, Siddiqui R, Anwar A, Shah MR, Khan NA. 2016. Gold nanoparticle conjugation enhances the antiacanthamoebic effects of chlorhexidine. Antimicrob. Agents Chemother. 60: 1283-1288.

5. Grace AN, Pandian K. 2007. Antibacterial efficacy of aminoglycosidic antibiotics protected gold nanoparticles-A brief study. Colloids Surfaces A: Physicochem. Eng. Aspects. 297: 63-70.

6. Wilczewska AZ, Niemirowicz K, Markiewicz KH, Car H. 2012. Nanoparticles as drug delivery systems. Pharmacol. Rep. 64: 1020-1037.

7. Martinez A, Visvesvara G. 1991. Laboratory diagnosis of pathogenic free-living amoebas: Naegleria, Acanthamoeba, and Leptomyxid. Clin. Lab Med. 11: 861-872.

8. Marciano-Cabral F, Cabral G. 2003. Acanthamoeba spp. as agents of disease in humans. Clin. Microbiol. Rev. 16: 273-307.

9. Khan NA. 2006. Acanthamoeba: biology and increasing importance in human health. FEMS Microbiol. Rev. 30: 564-595.

10. Seal D, Hay J, Kirkness C. 1995. Chlorhexidine or polyhexamethylene biguanide for Acanthamoeba keratitis. Lancet 345: 136.

11. Ishibashi $\mathrm{Y}$, Matsumoto $\mathrm{Y}$, Kabata $\mathrm{T}$, Watanabe $\mathrm{R}$, Hommura S, Yasuraoka K, et al. 1990. Oral itraconazole and topical miconazole with debridement for Acanthamoeba keratitis. Am. J. Ophthalmol. 109: 121-126.

12. Visvesvara GS, Moura H, Schuster FL. 2007. Pathogenic and opportunistic free-living amoebae: Acanthamoeba spp., Balamuthia mandrillaris, Naegleria fowleri, and Sappinia diploidea. FEMS Immunol. Med. Microbiol. 50: 1-26.

13. Girois S, Chapuis F, Decullier E, Revol B. 2005. Adverse effects of antifungal therapies in invasive fungal infections: review and meta-analysis. Eur. J. Clin. Microbiol. Infect. Dis. 24: 119-130.

14. Martín-Navarro CM, López-Arencibia A, Sifaoui I, ReyesBatlle M, Valladares B, Martínez-Carretero E, et al. 2015. Statins and voriconazole induce programmed cell death in Acanthamoeba castellanii. Antimicrob. Agents Chemother. 59: 2817-2824.

15. Thomson S, Rice CA, Zhang T, Edrada-Ebel R, Henriquez FL, Roberts CW. 2017. Characterisation of sterol biosynthesis and validation of $14 \alpha$-demethylase as a drug target in Acanthamoeba. Sci. Rep. 7: 8247. 
16. Anwar A, Siddiqui R, Hussain MA, Ahmed D, Shah MR, Khan NA. 2018. Silver nanoparticle conjugation affects antiacanthamoebic activities of amphotericin B, nystatin, and fluconazole. Parasitol. Res. 117: 265-271.

17. Anwar A, Shah MR, Muhammad SP, Afridi S, Ali K. 2016. Thiopyridinium capped silver nanoparticle based supramolecular recognition of $\mathrm{Cu}$ (I) in real samples and T-lymphocytes. New J. Chem. 40: 6480-6486.

18. Sissons J, Alsam S, Stins M, Rivas AO, Morales JL, Faull J, et al. 2006. Use of in vitro assays to determine effects of human serum on biological characteristics of Acanthamoeba castellanii. J. Clin. Microbiol. 44: 2595-2600.

19. Khan NA, Siddiqui R. 2009. Acanthamoeba affects the integrity of human brain microvascular endothelial cells and degrades the tight junction proteins. Int. J. Parasitol. 39: 1611-1616.

20. Ali SM, Siddiqui R, Ong SK, Shah MR, Anwar A, Heard PJ, et al. 2017. Identification and characterization of antibacterial compound (s) of cockroaches (Periplaneta americana). Appl. Microbiol. Biotechnol. 101: 253-286.

21. Radwan MA, AlQuadeib BT, Šiller L, Wright MC, Horrocks B. 2017. Oral administration of amphotericin B nanoparticles: antifungal activity, bioavailability and toxicity in rats. Drug Deliv. 24: 40-50.

22. Rodino S, Butu M, Negoescu C, Caunii A, Cristina R, Butnariu M. 2014. Spectrophotometric method for quantitative determination of nystatin antifungal agent in pharmaceutical formulations. Digest J. Nanomater. Biostruc. 9: 1215-1222.

23. Singh A, Sharma P, Majumdar D. 2011. Development and validation of different UV-spectrophotometric methods for the estimation of fluconazole in bulk and in solid dosage form. Indian J. Chem. Technol. 18: 357-362.

24. Suri SS, Fenniri H, Singh B. 2007. Nanotechnology-based drug delivery systems. J. Occup. Med. Toxicol. 2: 16.

25. Walsh MD, Hanna SK, Sen J, Rawal S, Cabral CB, Yurkovetskiy AV, et al. 2012. Pharmacokinetics and antitumor efficacy of XMT-1001, a novel, polymeric topoisomerase I inhibitor, in mice bearing HT-29 human colon carcinoma xenografts. Clin. Cancer Res. 18: 2591-2602.

26. Zazo H, Colino CI, Lanao JM. 2016. Current applications of nanoparticles in infectious diseases. J. Control Release. 224: 86-102.

27. Ahmed D, Anwar A, Khan AK, Ahmed A, Shah MR, Khan NA. 2017. Size selectivity in antibiofilm activity of 3-(diphenylphosphino) propanoic acid coated gold nanomaterials against Gram-positive Staphylococcus aureus and Streptococcus mutans. AMB Express 7: 210.

28. Dakal TC, Kumar A, Majumdar RS, Yadav V. 2016. Mechanistic basis of antimicrobial actions of silver nanoparticles. Front Microbiol. 7: 1831.

29. Pal S, Tak YK, Song JM. 2007. Does the antibacterial activity of silver nanoparticles depend on the shape of the nanoparticle? A study of the gram-negative bacterium Escherichia coli. Appl. Environ. Microbiol. 73: 1712-1720.

30. Zhao G, Stevens SE. 1998. Multiple parameters for the comprehensive evaluation of the susceptibility of Escherichia coli to the silver ion. Biometals 11: 27-32. 